
\title{
25 Research Soure \\ Differential Regulation of Intramuscular Fat and Abdominal Fat Deposition in Chickens
}

\section{Na Luo}

Institute of Animal Sciences, Chinese Academy of Agricultural Sciences

\section{Jingting Shu}

Poultry Institute, Chinese Academy of Agricultural Sciences

\section{Xiaoya Yuan}

Institute of Animal Sciences, Chinese Academy of Agricultural Sciences

Yuxi Jin

Institute of Animal Sciences, Chinese Academy of Agricultural Sciences

Huanxian Cui

Institute of Animal Sciences, Chinese Academy of Agricultural Sciences

\section{Guiping Zhao}

Institute of Animal Sciences, Chinese Academy of Agricultural Sciences

Jie Wen ( $\square$ wenjie@caas.cn )

Institute of Animal Sciences, Chinese Academy of Agricultural Sciences

\section{Research Article}

Keywords: intramuscular fat, abdominal fat, chicken, transcriptomics, WGCNA

Posted Date: March 1st, 2022

DOI: https://doi.org/10.21203/rs.3.rs-1312130/v1

License: (c) (1) This work is licensed under a Creative Commons Attribution 4.0 International License.

Read Full License

Version of Record: A version of this preprint was published at BMC Genomics on April 15th, 2022. See the published version at https://doi.org/10.1186/s12864-022-08538-0. 


\section{Abstract}

Background: Chicken intramuscular fat (IMF) content is closely related to meat quality and performance such as tenderness and flavor. Abdominal fat (AF) in chickens is one of the main waste products at slaughter. Excessive AF reduces feed efficiency and carcass quality.

Results: To analyze the differential deposition of IMF and AF in chickens, gene expression profiles in the breast muscle (BM) and AF tissues of 18 animals were analyzed by differential expression analysis and weighted co-expression network analysis. The results showed that IMF deposition in BM was associated with pyruvate and citric acid metabolism through GAPDH, LDHA, GPX1, GBE1, and other genes, whereas AF deposition was related to acetyl CoA and glycerol metabolism through FABP1, ELOVL6, SCD, ADIPOQ, and other genes. Carbohydrate metabolism plays an important role in IMF deposition, and fatty acid and glycerol metabolism regulate AF deposition.

Conclusion: In conclusion, this study elucidated the molecular mechanism governing IMF and AF deposition through key genes and signaling pathways and provided a theoretical basis for producing high-quality broilers.

\section{Introduction}

Fat deposition in broilers is affected by genetic factors (breed, sex, and genotype) and nongenetic factors (nutrition, age, and environmental factors). The rate of fat deposition varies across tissues in broilers. In addition, glucose and lipid metabolism and hormone sensitivity vary in adipocytes from different tissues [1-4]. The deposition of abdominal fat (AF) and intramuscular fat (IMF) is regulated by different mechanisms in chickens [5], and this characteristic allows the genetic selection of broilers with high IMF and low AF [6].

Previous studies showed a significant and positive correlation between IMF and AF [7, 8]. In contrast, a study compared two broiler breeds selected for AF percentage and found that the breed with lower AF had higher IMF. This discrepancy between studies may be caused by breeding characteristics, environmental factors, genetic selection, and sampling methods [6]. A balanced selection program (increasing IMF percentage while decreasing AF) was more effective than selecting for IMF alone in decreasing AF percentage in Jingxing yellow chicken [9]. Various genetic mechanisms regulate lipid deposition in tissues. Genes encoding perilipin and long-chain acyl CoA dehydrogenase are highly expressed in subcutaneous adipocytes, whereas genes encoding bone morphogenetic proteins 4 and 7 are abundantly expressed in intramuscular adipocytes [10]. Weighted correlation network analysis of transcriptome data showed that the transcription factor CREB3L 1 was associated with low AF, whereas the transcription factor $L 3 M B T L 1$ and the cofactor TNIP1 were related to high IMF and low AF in the Jingxing-Huang chicken line [11]. These results underscore the need to study the genetic mechanisms regulating fat deposition in broiler chickens. 
Few studies jointly evaluated IMF and AF in broilers. This study evaluates fat differential deposition in breast muscle (BM) and AF tissues, gene expression profiles these tissues based on transcriptome data, and genes and signaling pathways involved in IMF and AF deposition in chickens, to reveal the difference of molecular regulation mechanisms.

\section{Results}

\section{Evaluation of sequencing data}

Transcriptomic data were obtained from the BM and AF of 18 broiler chickens. Thirty-six RNA-sequencing (RNA-seq) libraries were constructed and sequenced (Additional file: Table S1). A total of 23,910 genes were detected, of which 17,179 genes were expressed in BM, and 18,912 genes were expressed in AF. In addition, 72.24\% (16753/23910) of the genes were expressed in both BM and AF (Fig. 1). Raw sequence reads from each library are shown in Additional file: Tables S2 and S3. The most genes abundantly expressed in BM and AF were glyceraldehyde-3-phosphate dehydrogenase and fatty acid-binding protein (FABP4), respectively.

To explore differences in IMF and AF deposition, Principal component analysis (PCA) of fatty acid composition (Additional file: Table S4) was performed to assess differences in fat deposition in BM and AF tissue (Fig. 2A) [12].

PCA analysis was performed on 16753 genes co-expressed in BM and AF (Fig. 2B). PCA analysis was performed on all expressed genes in $\mathrm{BM}$ and $\mathrm{AF}$, respectively (Fig. 2C and 2D). As expected, there was a strong separation between the two tissues.

\section{WGCNA identified module genes significantly related to phenotypes}

Genes with fragments per kilobase of transcript per million fragments mapped values of less than 1 were excluded. Gene co-expression networks were constructed using 18,280 DEGs from BM and 19,766 DEGs from AF, and the coordination matrix was converted to a topology matrix. The similarity in gene coexpression between modules was quantified using a dynamic tree-cutting algorithm, and modules with height values of less than 0.25 were merged (the minimum number of genes in the module was set to 30 ) (Fig. 3).

Twenty and twenty-seven expression modules were detected in BM (Additional file: Table S5) and AF (Additional file: Table S6), respectively (Fig. 4), and the number of genes in each network module was counted. The largest module in BM (blue) and AF (turquoise) contained 6972 and 5755 genes, respectively, and the smallest module in BM (dark brown) and AF (white) contained 113 and 47 genes, respectively. 
Gene modules significantly associated with genetic traits were identified. Absolute values of 0 and 1 indicated weak and strong correlation between the module and the trait, respectively. Among the evaluated traits, IMF was significantly and positively correlated with the blue module $(r=0.51, P=0.04)$, triglycerides (TG) was significantly and positively associated with the darkred module $(r=0.72, P=0.002)$, total cholesterol (TCHO) was significantly and positively related to the darkorange module $(r=0.63, P=$ 0.008), phospholipids (PLIP) was significantly and positively associated with the orange and blue modules $(r=-0.64, P=0.007 ; r=-0.51, P=0.04)$ and traits such as fatty acids composition showed significant correlations with related modules. AF weight showed a significant and positive correlation with the darkgreen and lightgreen modules $(r=0.65, P=0.009 ; r=0.71, P=0.003)$, and AF percentage showed a significant and positive association with the lightgreen and yellow modules $(r=0.64, P=0.01 ; r$ $=0.54, P=0.04)$. In AF tissue, TG was significantly and positively correlated with the darkred and turquoise modules $(r=0.55, P=0.03 ; r=0.57, P=0.03)$. TCHO showed a significant and positive association with the brown and blue modules $(r=0.87, P=3 e-05 ; r=0.64, P=0.01)$. PLIP was significantly and positively related to the grey60 module $(r=0.91, P=3 e-06)$, and traits such as fatty acids composition showed a significant and positive correlations with related modules.

\section{Identification of DEGs in BM and AF}

Analysis using Deseq2 detected 461 DEGs (163 upregulated and 298 downregulated) in the high and low IMF groups (Additional file: Table S7). Pathway enrichment analysis revealed that these genes were enriched in 29 pathways, mainly including metabolic pathways, MAPK signaling pathways, arachidonic acid metabolism, glycolysis/gluconeogenesis, starch, and sucrose metabolism, fructose and mannose metabolism, and other signaling pathways (Additional file: Table S8). GO analysis showed that DEGs in this group were enriched in 27 biological processes, 16 cellular components, and 12 molecular functions (Additional file: Table S9). 2 A total of 2010 genes (1301 upregulated and 700 downregulated) were detected in the high and low AF groups (Additional file: Table S10). These genes were enriched in 59 KEGG pathways, mainly including metabolic pathways, PPAR signaling pathway, MAPK signaling pathway, tyrosine metabolism, glycerolipid metabolism, phenylalanine metabolism, glycerophospholipid metabolism, pyruvate metabolism, unsaturated fatty acid biosynthesis, fatty acid metabolism, and other signaling pathways (Additional file: Table S11). GO analysis showed that DEGs in this group were enriched in 66 biological processes, 21 cellular components, and 18 molecular functions (Additional file: Table S12).

\section{Identification of pathways in BM and AF}

The genes expressed in the modules significantly associated with all phenotypes were selected from BM and AF tissues, respectively. The significantly expressed genes in the modules significantly associated with the traits were pooled, and a total of 3818 expressed genes were identified in BM tissue, while a total of 5826 expressed genes were identified in AF tissue.

These genes were analyzed separately in association with differentially expressed genes, in which 114 co-expressed genes were obtained in BM tissue (Fig. 5A), while a total of 1229 co-expressed genes were 
obtained in AF tissue (Fig. 5B).

The differential module genes obtained from the two tissues were analyzed separately for KEGG pathway enrichment. In BM tissue, 114 genes were assigned to 5 KEGG pathways $(P<0.05)$ (Fig. 6A), and genes were enriched in MAPK signaling pathway, ECM-receptor interaction, Gap junction, Tight junction, Vascular smooth muscle contraction, GnRH signaling pathway, and Focal adhesion. In AF tissue, 1229 genes were assigned to $41 \mathrm{KEGG}$ pathways $(P<0.05)$ (Fig. 6B), and genes were mainly enriched in biological processes, including Oxidative phosphorylation, $A B C$ transporters, $C$-type lectin receptor signaling pathway, Phosphatidylinositol signaling system.

\section{Identification of co-expressed genes}

The molecular expression networks between pathway and co-expressed genes in BM (Fig. 7A) and AF (Fig. 7B) were performed, respectively. The genes more abundantly expressed in BM with high IMF were related to muscle development (TPM2, MTMR7) and glycolipid metabolism (LDHA, AMY1A, ST3GAL6, GBE1, SGPL1, GPX1, COX5A, ALDH5A1). The genes more significantly expressed in the high AF group were related to fatty acid metabolism (ELOVL6, SCD, FABP1, ME3, ADIPOQ, HMGCS2, PDGFA, ACAT2).

\section{Discussion}

Yellow feather broiler chickens are widely consumed in China, and chicken meat production has increased in recent years. IMF and AF contents strongly affect the quality of chicken meat. Although the mechanisms underlying fat deposition in broiler chickens are well known, differences in the rate of fat deposition in BM and AF tissues have not been determined. A large number of DEGs can be identified by RNA-seq analysis; however, the characterization of gene expression patterns and correlation with phenotypes are challenging, underscoring the need to perform weighted co-expression network analyses. In the study, DEGs were identified in BM and AF by RNA-seq analysis, and joint analysis with WGCNA to identify specifically expressed genes and signaling pathways for IMF and AF deposition. The molecular regulatory mechanisms of fat deposition in different tissues at the same age were elucidated in chicken.

Based on the differentially expressed genes, the results of KEGG signaling pathway analysis showed that DEGs from BM and AF tissues were jointly enriched in the AGE-RAGE signaling pathway, amino acid biosynthesis, cytokine-cytokine receptor interactions, local adhesion, GnRH signaling pathway, MAPK signaling pathway, metabolic pathway, in diabetic complications. tight junctions and other signaling pathways. Studies have shown that MAPK signaling pathway is involved in lipid deposition [13, 14], which can regulate PPAR pathway[15]. Moreover, PPAR signaling pathway also has the function of regulating lipid metabolism $[16,17]$.

In contrast, DEGs in BM tissue were specifically enriched in pathways related to gluconeogenesis (glycolysis/gluconeogenesis, starch, sucrose metabolism, fructose, mannose metabolism, pentose, and glucuronide). $\angle D H A$ and $\angle D H B$ in the glycolysis/gluconeogenesis signaling pathway are involved in pyruvate metabolism and tricarboxylic acid cycle, which can facilitate the glycolytic process by 
converting pyruvate to lactate [18]. In addition, GBE1 is essential in starch and glycogen formation metabolism, and there is a strong link between the hexokinase family genes $H K 1, H K 2, H K 3$ in this pathway and the metabolic process of glucose. These data suggest that IMF deposition in BM tissue is mainly dependent on glucose metabolism, with some energy metabolism involved in the process.

DEGs in BM tissue were specifically enriched in pathways related to fatty acid syntheses (PPAR signaling, glycerolipid metabolism, glycerophospholipid metabolism, and unsaturated fatty acid biosynthesis). Sixteen genes were enriched in the PPAR signaling pathway, including HMGCS2 (catalyze ketogenesis) [19, 20], ACOX1 (lipid degradation)[21], ADIPOQ (adipocyte differentiation)[22], APOA1 and ME3 (cholesterol metabolism)[23], $S C D$ (fatty acid transporter proteins)[24], PLIN1 and PLIN2 (lipid droplet protection)[25-27], ACSL 1[28], FABP7, FABP1, FADS2. The formation of intracellular lipid droplets is a highly conserved process, including fatty acid transport and activation, neutral lipid synthesis, and lipid droplet formation, which is regulated by many factors and pathways[29]. These results suggest that AF deposition is dependent on fatty acid synthesis and transport and lipid droplet formation.

WGCNA was performed using RNA-seq data to identify expressed genes in different modules and predict the role of genes in lipid deposition. Several modules were significantly associated with IMF/AFW/AFP, lipid composition, and fatty acids metabolism in BM and AF, and the expressed genes significantly associated with the phenotypes in the modules were aggregated together and analyzed jointly with DEGs, which could further identify the functional genes that play important roles in the IMF and AF deposition.

We identified 114 expressed genes associated with Metabolic pathways, Starch and sucrose metabolism, Intestinal immune network for IgA production, Adrenergic signaling in cardiomyocytes, and Glycolysis/Gluconeogenesis in BM. These pathways are regulated by genes such as ALDH5A1, LDHA, $G A P D H, G B E 1$, and GPX1 and may play roles in IMF deposition. A total of 1229 expressed genes associated with 41 signaling pathways were identified in $A F$, of which 20 pathways that may play roles in AF deposition via PPAR signaling pathway, amino acid biosynthesis, oxidative phosphorylation, may be controlled by several genes, including ADIPOQ, ELOVL6, HMGCS2, ME3, DGKE, AOX1, UBB, SCD, APOC3, and $F A B P 1$. The results revealed that IMF deposition in BM tissue was regulated by gluconeogenesisrelated pathways (glycolysis/gluconeogenesis, starch and, sucrose metabolism signaling pathways), and by several genes, including GAPDH, $L D H A, G P X 1, A L D H 5 A 1$, and GBE1, among which, the key differential gene $A L D H 5 A 1$ can catalyze a step in the degradation of the inhibitory neurotransmitter $y$-aminobutyric acid [30]. T The protein encoded by $L D H A$ is involved in pyruvate metabolism by catalyzing the conversion of lactate to pyruvate in anaerobic glycolysis. GAPDH plays an important role in glycerol metabolism. These data further suggest that IMF deposition in BM is dependent on gluconeogenesis and energy metabolism.

While expressed genes in AF tissue are enriched to PPAR signaling pathway, oxidative phosphorylation, amino acid biosynthesis, and other signaling pathways, acting through genes such as FABP1, ELOVL6, $S C D$, and $A D I P O Q$. PPAR signaling pathway is involved in lipid droplets formation and mitochondrial metabolism, and fatty acid oxidation and lipid synthesis are necessary for cellular signaling [31]. FABP1 
plays a crucial role in the PPAR pathway, PPARG expression, as well as fatty acid uptake, transport, and metabolism in vivo[32]. In addition, $P P A R Y$ can be regulated by modulating $S C D 1$ expression to control fatty acid synthesis in adipocytes [33]. ELOVLs encode long-chain and extra-long-chain fatty acid elongases that play an important role in the fatty acid synthesis and can limit fatty acid elongation, and ELOVL6 is involved in the synthesis of fatty acid enzymes in vivo, promoting fatty acid elongation. ACAT2 encodes acetyl-coenzyme $A$ acetyltransferase 2 , which is involved in acetyl-coenzyme A metabolism. These results suggest that AF deposition may result from changes in fatty acid synthesis through mitochondrial activity.

The above results suggest that different signaling pathways regulate fat deposition in $\mathrm{BM}$ and $\mathrm{AF}$ tissues. IMF deposition in BM was associated with pyruvate and citrate metabolism through GAPDH, $\angle D H A, G P X 1, G B E 1$, and other genes, whereas AF deposition was related to acetyl-coenzyme $\mathrm{A}$ and glycerophospholipid metabolism through FABP1, ELOVL6, SCD, and ADIPOQ. The transcriptional regulation of genes in network modules associated with traits and metabolic pathway analysis can provide new insights into the genetic mechanisms governing fat deposition in broiler chickens.

\section{Conclusion}

The analysis of transcriptome data from $B M$ and $A F$, combined with differential expression analysis and WGCNA, showed that the genes GAPDH, LDHA, GPX1, and GBE1 were involved in regulating IMF deposition, and the genes FABP1, ELOVL6, SCD, and ADIPOQ determined AF deposition.

Glycolysis/gluconeogenesis and other signaling pathways play an important role in IMF deposition, whereas PPAR metabolism controls AF deposition. Therefore, we hypothesized that IMF deposition in BM may energy metabolism during myocyte gluconeogenesis, while fatty acid synthesis pathways may affect AF deposition.

\section{Materials And Methods}

\section{Animals and Sample Collection}

Eighteen Wenchang broiler chickens of similar age were selected. The animals were reared in an environmentally-controlled room with three-tier cages in the animal facility of the Institute of Poultry Research of the Chinese Academy of Agricultural Sciences (Yangzhou, China). The diet met the requirements of the National Resources Committee (1994) and the Standards for Chicken Feeding (2004) established by the Ministry of Agriculture (Beijing, China). At 98 days, after a 12-hour fast, the animals were stunned by electric shock and killed by cervical dislocation. After slaughter, breast muscle (BM) and AF were collected and immediately stored at $-80^{\circ} \mathrm{C}$ until use.

\section{Measurement of Biochemical Indices[12]}

The determination of fatty acids in BM and AF tissues refers to the national standard $\mathrm{GB} / \mathrm{T}$ 5413.272010 Food Safety National Standard for infant food and dairy products determination of fatty acids $\square$ in 
the first method: acetyl chloride - methanol methyl esterification method. The determination of intramuscular fat in BM tissue refers to the national standard GGB/T 5009.6-2016 National Standard for Food Safety determination of fat in foodlin the first method: Soxhlet extraction. The TG, TCHO, and PLIP contents in BM and AF tissues were measured using assay kits (Nanjing Jiancheng Bioengineering Institute, Nanjing, China, and Beijing Leichuang Biotechnology Co., LTD, China). We weighed $2.0 \pm 0.01 \mathrm{~g}$ breast muscle and abdominal fat samples were mixed with $18 \mathrm{~mL}$ anhydrous ethanol and placed in a 50 $\mathrm{ml}$ centrifuge tube. Each sample was homogenized by a hand-held high-speed homogenizer $(35,000$ $\mathrm{r} / \mathrm{min}, 15 \mathrm{~s} /$ time). The samples were crushed, centrifuged at $2500 \mathrm{r} / \mathrm{min}$ for $10 \mathrm{~min}$, After centrifugation, the supernatant was used for measurement. Refer to the instruction for specific sample loading quantity. After incubation, the absorbance value $(510 \mathrm{~nm})$ was measured by using a microplate reader (Tecan Infinite 200 Pro, Switzerland).

\section{Transcriptome Profiling}

Transcriptome profiling of 18 breast muscle tissues and 18 abdominal fat tissues was carried out using an Illumina PE150 platform (Berry Genomics; Beijing, China) (https://www.berrygenomics.com). RNA was extracted as described previously [34]. Adapter sequences and other low-quality data were removed using Cutadapt. Reads were aligned to the reference genome using HISAT, while clean reads obtained by filtering were compared to the reference genome according to the gene position information specified in the genome annotation file gtf, and the total comparison rate was approximately $90 \%$.

\section{Differential Expression Analysis}

Based on phenotypic data, DEGs were identified in BM tissue with high IMF and low IMF (from three animals each) and AF tissue with high AF and low AF (from three animals each) using DESeq2 in R [35]. Adjusted p-values (q-values) were calculated using Benjamini and Hochberg's approach for controlling the false discovery rate. Genes with $\mid \log 2$ (fold change) $\mid \geq 0.58$ and $P<0.05$ were considered differentially expressed.

\section{Weighted Gene Co-expression Network Analysis (WGCNA)}

WGCNA was performed using the WGCNA package [36] with default settings and minor modifications. In BM analysis ( $n=18$ ), the minModuleSize was set to 50 , and mergeCutHeight was set to 0.25 for tissue- or stage-specific module detection (soft threshold $=10)$. In AF analysis $(n=18)$, the minModuleSize was set to 50 , and mergeCutHeight was set to 0.2 for tissue- or stage-specific module detection (soft threshold = 6). Dendrograms were created using topology overlap matrix, module detection, and similar module merging functions (minModuleSize $=30$ ). Module-trait relationships were calculated, and correlations were adjusted using Benjamini-Hochberg correction.

\section{Pathway Enrichment Analyses}

Gene ontology (GO) enrichment analysis was performed using the DAVID functional annotation clustering tool to identify gene classes and categories. Kyoto Encyclopedia of Genes and Genomes 
(KEGG) pathway enrichment analysis was performed using KOBAS version 3.0 (http://kobas.cbi.pku.edu.cn). The significance level was set at $P<0.05$.

\section{Statistical Analyses}

The significance of differences between means was assessed using Student's t-test in SPSS version 22.0 (IBM Corp, Armonk, NY, USA). P-values less than 0.05 were considered statistically significant. Data are presented as the mean \pm standard error of the mean.

\section{Abbreviations}

ACAT2: Acetyl-CoA Acetyltransferase 2; ACOX1: Acyl-CoA Oxidase 1; ACSL1: Acyl-CoA Synthetase Long Chain Family Member 1; ADIPOQ: Adiponectin, C1Q And Collagen Domain Containing; AF: Abdominal fat; AFW: Abdominal fat weight; AFP: Abdominal fat percentage;ALDH5A1: Aldehyde Dehydrogenase 5 Family Member A1; AMY1A: Amylase Alpha 1A; AOX1: Aldehyde Oxidase 1; APOA1: Apolipoprotein A1; APOC3: Apolipoprotein C3; BM: Breast Muscle; COX5A: Cytochrome C Oxidase Subunit 5A; CREB3L1: CAMP Responsive Element Binding Protein 3 Like 1; DEGs: Differential Expressed Genes; DGKE: Diacylglycerol Kinase Epsilon; ECM: Extracellular Matrix; ELOVL6: ELOVL Fatty Acid Elongase 6; FABP1: Fatty Acid Binding Protein 1; FABP4: Fatty Acid Binding Protein 4; FABP7: Fatty Acid Binding Protein 7; FADS2: Fatty Acid Desaturase 2; GAPDH: Glyceraldehyde-3-Phosphate Dehydrogenase; GBE1: 1,4-AlphaGlucan Branching Enzyme 1; GO: Gene Ontology; GPX1: Glutathione Peroxidase 1; HK1: Hexokinase 1; HK2: Hexokinase 2; HK3: Hexokinase 3; HMGCS2: 3-Hydroxy-3-Methylglutaryl-CoA Synthase 2; IMF: Intramuscular Fat; KEGG: Kyoto Encyclopedia of Genes and Genomes; L3MBTL1: Histone Methyl-Lysine Binding Protein 1; LDHA: Lactate Dehydrogenase A; ME3: Malic Enzyme 3; PCA: Principal Component Analysis; PDGFA: Platelet Derived Growth Factor Subunit A; PLIN1: Perilipin 1; PLIN2: Perilipin 2; PLIP: Phospholipid; RNA-Seq: RNA-sequencing; SCD: Stearoyl-CoA Desaturase; SGPL1: Sphingosine-1Phosphate Lyase 1; ST3GAL6: ST3 Beta-Galactoside Alpha-2,3-Sialyltransferase 6; TCHO: Total Cholesterol; TG: Triglycerides; TNP1: Transition Protein 1; UBB: Ubiquitin B; WGCNA: Weighted Gene Coexpression Network Analysis.

\section{Declarations}

\section{Ethics approval and consent to participate}

This study was conducted in accordance with the Guide for the Care and Use of Laboratory Animals published by the Ministry of Science and Technology (Beijing, China). The study was approved by the Research Ethics Committee of the Institute of Animal Sciences of the Chinese Academy of Agricultural Sciences (Beijing, China) (Protocol No. IAS2019-21), all methods were carried out in accordance with relevant guidelines and regulations. This study was carried out in compliance with the ARRIVE guidelines (Animal Research: Reporting of In Vivo Experiments). 
Not applicable.

\section{Availability of data and materials}

The RNA sequencing raw data reported in this paper have been deposited in the Genome Sequence Archive[37] in BIG Data Center[38] under accession number CRA006031 which can be publicly accessed at http://bigd.big.ac.cn/gsa. All data analyzed during this study are included in this article and its additional file.

\section{Competing interests}

The authors declare that they have no competing interests.

\section{Funding}

The research was funded by grants from the National Natural Science Foundation of China (32172723), the State Key Laboratory of Animal Nutrition (2004DA125184G2109), the Agricultural Science and Technology Innovation Program (ASTIP-IAS04), and the Earmarked Fund for Modern Agro-Industry Technology Research System (CARS-41).

\section{Authors' contributions}

LN and SJT performed the study, analyzed the data, and drafted the manuscript. YXY and JYX collected samples and analyzed the data. HXC contributed to the design of the study, acquiring the funding, and modifying the manuscript. GPZ contributed to providing resources and contributed to acquiring the funding. JW designed the study and followed up on the overall project. All authors submitted comments on drafts, and read and approved the final manuscript.

\section{Acknowledgments}

Not applicable.

\section{References}

1. Chu W, Wei W, Han H, Gao Y, Liu K, Tian Y, Jiang Z, Zhang L, Chen J: Muscle-specific downregulation of GR levels inhibits adipogenesis in porcine intramuscular adipocyte tissue. Sci Rep. 2017; 7(1):510.

2. Han H, Wei W, Chu W, Liu K, Tian Y, Jiang Z, Chen J: Muscle Conditional Medium Reduces Intramuscular Adipocyte Differentiation and Lipid Accumulation through Regulating Insulin Signaling. Int J Mol Sci. 2017; 18(8).

3. Smith SB, Crouse JD: Relative contributions of acetate, lactate and glucose to lipogenesis in bovine intramuscular and subcutaneous adipose tissue. J Nutr. 1984; 114(4):792-800.

4. Wang S, Zhou G, Shu G, Wang L, Zhu X, Gao P, Xi Q, Zhang Y, Yuan L, Jiang Q: Glucose utilization, lipid metabolism and BMP-Smad signaling pathway of porcine intramuscular preadipocytes 
compared with subcutaneous preadipocytes. Cell Physiol Biochem. 2013; 31(6):981-996.

5. Zhang M, Li F, Ma XF, Li WT, Jiang RR, Han RL, Li GX, Wang YB, Li ZY, Tian YD et al: Identification of differentially expressed genes and pathways between intramuscular and abdominal fat-derived preadipocyte differentiation of chickens in vitro. BMC Genomics. 2019; 20(1):743.

6. Leng L, Zhang H, Dong JQ, Wang ZP, Zhang XY, Wang SZ, Cao ZP, Li YM, Li H: Selection against abdominal fat percentage may increase intramuscular fat content in broilers. J Anim Breed Genet. 2016; $133(5): 422-428$.

7. Chen JL, Zhao GP, Zheng MQ, Wen J, Yang N: Estimation of genetic parameters for contents of intramuscular fat and inosine-5'-monophosphate and carcass traits in Chinese Beijing-You chickens. Poult Sci. 2008; 87(6):1098-1104.

8. Ge K, Ye P, Yang L, Kuang J, Chen X, Geng Z: Comparison of slaughter performance, meat traits, serum lipid parameters and fat tissue between Chaohu ducks with high- and low-intramuscular fat content. Anim Biotechnol. 2020; 31(3):245-255.

9. Jiang M, Fan WL, Xing SY, Wang J, Li P, Liu RR, Li QH, Zheng MQ, Cui HX, Wen J et al: Effects of balanced selection for intramuscular fat and abdominal fat percentage and estimates of genetic parameters. Poult Sci. 2017; 96(2):282-287.

10. Zhou G, Wang S, Wang Z, Zhu X, Shu G, Liao W, Yu K, Gao P, Xi Q, Wang X et al: Global comparison of gene expression profiles between intramuscular and subcutaneous adipocytes of neonatal landrace pig using microarray. Meat Sci. 2010; 86(2):440-450.

11. Xing S, Liu R, Zhao G, Liu L, Groenen MAM, Madsen O, Zheng M, Yang X, Crooijmans R, Wen J: RNASeq Analysis Reveals Hub Genes Involved in Chicken Intramuscular Fat and Abdominal Fat Deposition During Development. Front Genet. 2020; 11:1009.

12. Na L, Huanxian C, Xiaoya Y, Yuxi J, Jingting S, Guiping Z, Jie W: Study on the Differences in the Composition of Intramuscular Fat and Abdominal Fat in Wenchang Chicken. China Poultry. 2021; 43(09):6-11.

13. Shen Y, Zhao Y, Zheng D, Chang X, Ju S, Guo L: Effects of orexin A on GLUT4 expression and lipid content via MAPK signaling in 3T3-L1 adipocytes. J Steroid Biochem Mol Biol. 2013; 138:376-383.

14. Gubern A, Barceló-Torns M, Barneda D, López JM, Masgrau R, Picatoste F, Chalfant CE, Balsinde J, Balboa MA, Claro E: JNK and ceramide kinase govern the biogenesis of lipid droplets through activation of group IVA phospholipase A2. J Biol Chem. 2009; 284(47):32359-32369.

15. Capobianco E, Martínez N, Fornes D, Higa R, Di Marco I, Basualdo MN, Faingold MC, Jawerbaum A: PPAR activation as a regulator of lipid metabolism, nitric oxide production and lipid peroxidation in the placenta from type 2 diabetic patients. Mol Cell Endocrinol. 2013; 377(1-2):7-15.

16. Moreno M, Lombardi A, Silvestri E, Senese R, Cioffi F, Goglia F, Lanni A, de Lange P: PPARs: Nuclear Receptors Controlled by, and Controlling, Nutrient Handling through Nuclear and Cytosolic Signaling. PPAR Res. 2010; 2010.

17. Cui HX, Liu RR, Zhao GP, Zheng MQ, Chen JL, Wen J: Identification of differentially expressed genes and pathways for intramuscular fat deposition in pectoralis major tissues of fast-and slow-growing 
chickens. BMC Genomics. 2012; 13:213.

18. Feng Y, Xiong Y, Qiao T, Li X, Jia L, Han Y: Lactate dehydrogenase A: A key player in carcinogenesis and potential target in cancer therapy. Cancer Med. 2018; 7(12):6124-6136.

19. Shukla SK, Liu W, Sikder K, Addya S, Sarkar A, Wei Y, Rafiq K: HMGCS2 is a key ketogenic enzyme potentially involved in type 1 diabetes with high cardiovascular risk. Sci Rep. 2017; 7(1):4590.

20. Lee YE, He HL, Shiue YL, Lee SW, Lin LC, Wu TF, Chang IW, Lee HH, Li CF: The prognostic impact of lipid biosynthesis-associated markers, HSD17B2 and HMGCS2, in rectal cancer treated with neoadjuvant concurrent chemoradiotherapy. Tumour Biol. 2015; 36(10):7675-7683.

21. He A, Dean JM, Lu D, Chen Y, Lodhi IJ: Hepatic peroxisomal $\beta$-oxidation suppresses lipophagy via RPTOR acetylation and MTOR activation. Autophagy. 2020; 16(9):1727-1728.

22. Gao Y, Li F, Zhang Y, Dai L, Jiang H, Liu H, Zhang S, Chen C, Zhang J: Silencing of ADIPOQ efficiently suppresses preadipocyte differentiation in porcine. Cell Physiol Biochem. 2013; 31(2-3):452-461.

23. Ray A, Ghosh A, Chakraborty R, Upadhyay SK, Maiti S, Sengupta S, Thukral L: Specific Cholesterol Binding Drives Drastic Structural Alterations in Apolipoprotein A1. J Phys Chem Lett. 2018; $9(20): 6060-6065$.

24. Jin S, Lee SH, Lee DH, Manjula P, Lee SH, Lee JH: Genetic association of DEGS1, ELOVL6, FABP3, FABP4, FASN and SCD genes with fatty acid composition in breast and thigh muscles of Korean native chicken. Anim Genet. 2020; 51(2):344-345.

25. MacPherson RE, Ramos SV, Vandenboom R, Roy BD, Peters SJ: Skeletal muscle PLIN proteins, ATGL and CGI-58, interactions at rest and following stimulated contraction. Am J Physiol Regul Integr Comp Physiol. 2013; 304(8):R644-650.

26. Feng YZ, Lund J, Li Y, Knabenes IK, Bakke SS, Kase ET, Lee YK, Kimmel AR, Thoresen GH, Rustan AC et al: Loss of perilipin 2 in cultured myotubes enhances lipolysis and redirects the metabolic energy balance from glucose oxidation towards fatty acid oxidation. J Lipid Res. 2017; 58(11):2147-2161.

27. Bosma M, Hesselink MK, Sparks LM, Timmers S, Ferraz MJ, Mattijssen F, van Beurden D, Schaart G, de Baets $\mathrm{MH}$, Verheyen FK et al: Perilipin 2 improves insulin sensitivity in skeletal muscle despite elevated intramuscular lipid levels. Diabetes. 2012; 61(11):2679-2690.

28. Tian W, Wang D, Wang Z, Jiang K, Li Z, Tian Y, Kang X, Liu X, Li H: Evolution, expression profile, and regulatory characteristics of ACSL gene family in chicken (Gallus gallus). Gene. 2021; 764:145094.

29. Walther TC, Chung J, Farese RV, Jr.: Lipid Droplet Biogenesis. Annu Rev Cell Dev Biol. 2017; 33:491510.

30. Escalera GI, Ferrer I, Marina LC, Sala PR, Salomons GS, Jakobs C, Pérez-Cerdá C: [Succinic semialdehyde dehydrogenase deficiency: decrease in 4-OH-butyric acid levels with low doses of vigabatrin]. An Pediatr (Barc). 2010; 72(2):128-132.

31. Lodhi IJ, Semenkovich CF: Peroxisomes: a nexus for lipid metabolism and cellular signaling. Cell Metab. 2014; 19(3):380-392. 
32. Furuhashi M, Hotamisligil GS: Fatty acid-binding proteins: role in metabolic diseases and potential as drug targets. Nature Reviews Drug Discovery. 2008; 7(6):489-503.

33. Risérus U, Tan GD, Fielding BA, Neville MJ, Currie J, Savage DB, Chatterjee VK, Frayn KN, O'Rahilly S, Karpe F: Rosiglitazone increases indexes of stearoyl-CoA desaturase activity in humans: link to insulin sensitization and the role of dominant-negative mutation in peroxisome proliferator-activated receptor-gamma. Diabetes. 2005; 54(5):1379-1384.

34. Liu L, Liu X, Cui H, Liu R, Zhao G, Wen J: Transcriptional insights into key genes and pathways controlling muscle lipid metabolism in broiler chickens. BMC Genomics. 2019; 20(1):863.

35. Love MI, Huber W, Anders S: Moderated estimation of fold change and dispersion for RNA-seq data with DESeq2. Genome Biol. 2014; 15(12):550.

36. Langfelder P, Horvath S: WGCNA: an R package for weighted correlation network analysis. BMC Bioinformatics. 2008; 9:559.

37. Wang Y, Song F, Zhu J, Zhang S, Yang Y, Chen T, Tang B, Dong L, Ding N, Zhang Q et al: GSA: Genome Sequence Archive<sup/>. Genomics Proteomics Bioinformatics. 2017; 15(1):14-18.

38. Database Resources of the BIG Data Center in 2019. Nucleic Acids Res. 2019; 47(D1):D8-d14.

\section{Figures}




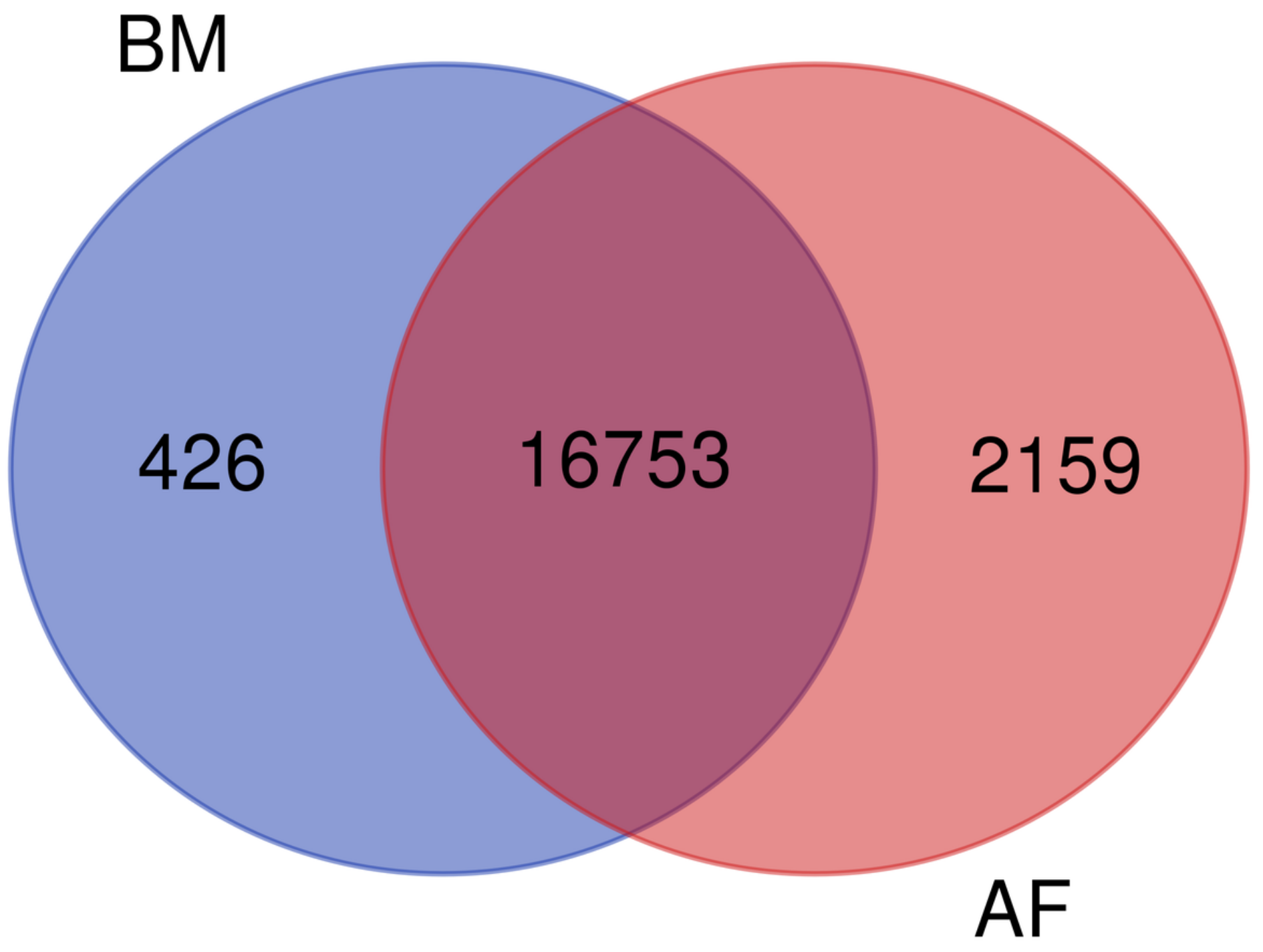

Figure 1

Venn diagrams of genes differentially expressed in the breast muscle and abdominal fat of broiler chickens. 

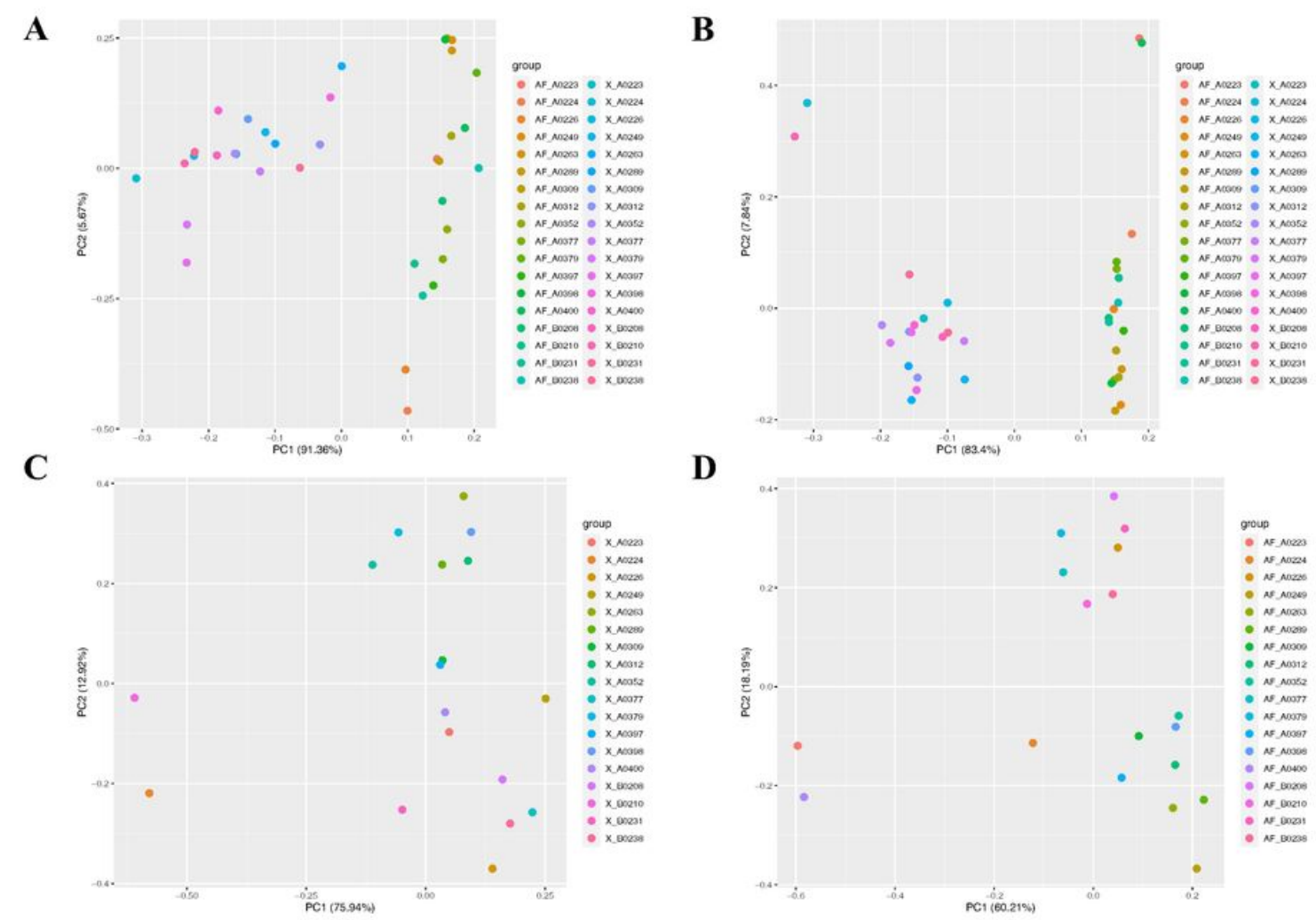

D

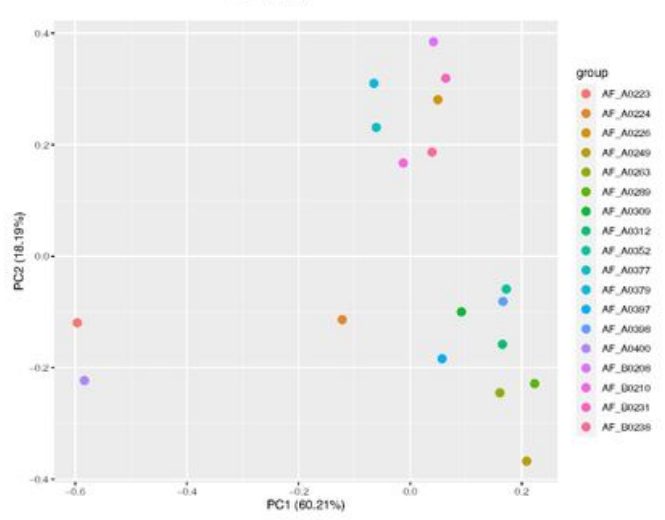

Figure 2

Principal component analysis (PCA) results of BM and AF tissue. (A) PCA of phenotypes in BM and AF. (B) PCA of genes expressed in both BM and AF. (C) PCA of genes expressed in BM. (D) PCA of genes expressed in AF.
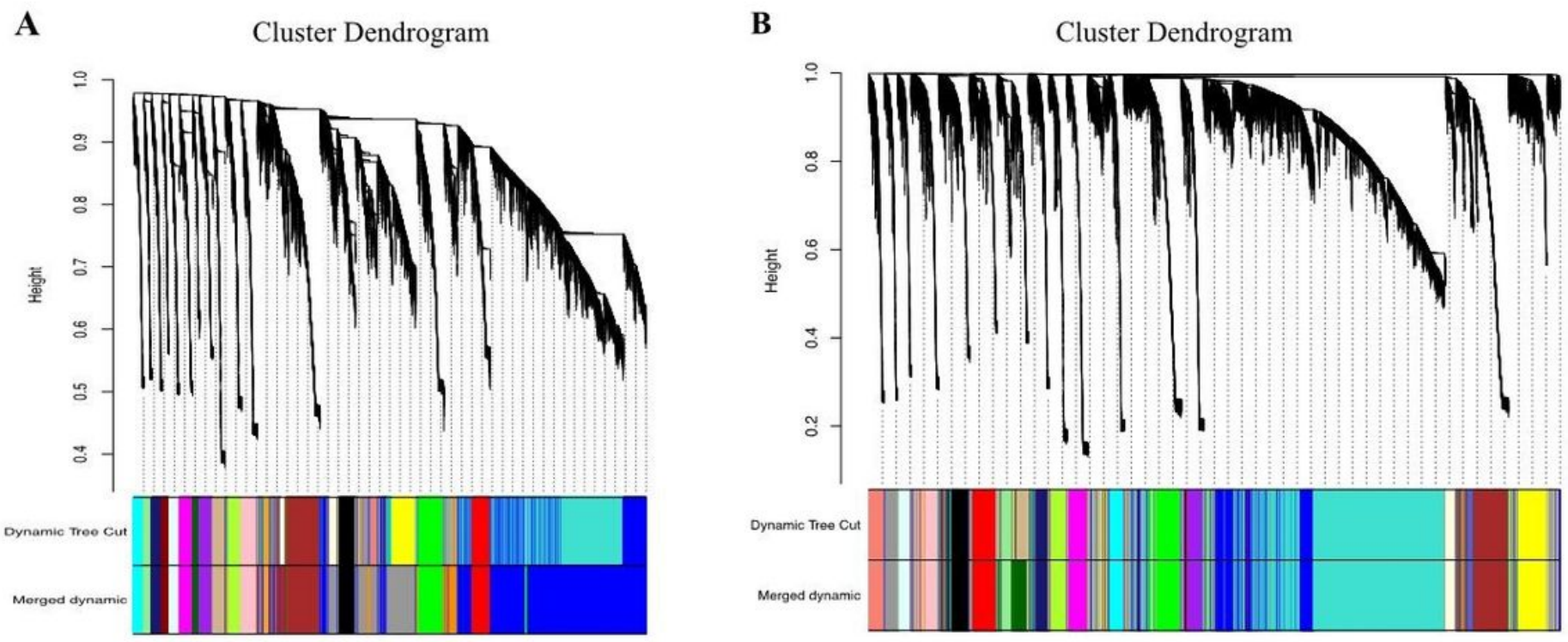

Figure 3 
Clustering of gene modules. (A) Breast muscle tissue microarray. (B) Abdominal fat tissue microarray. Upper panel: genes were clustered into different groups. Lower panel: genes were assigned to modules after dynamic tree-cutting and merging.

A

Module-stage-trait relationships

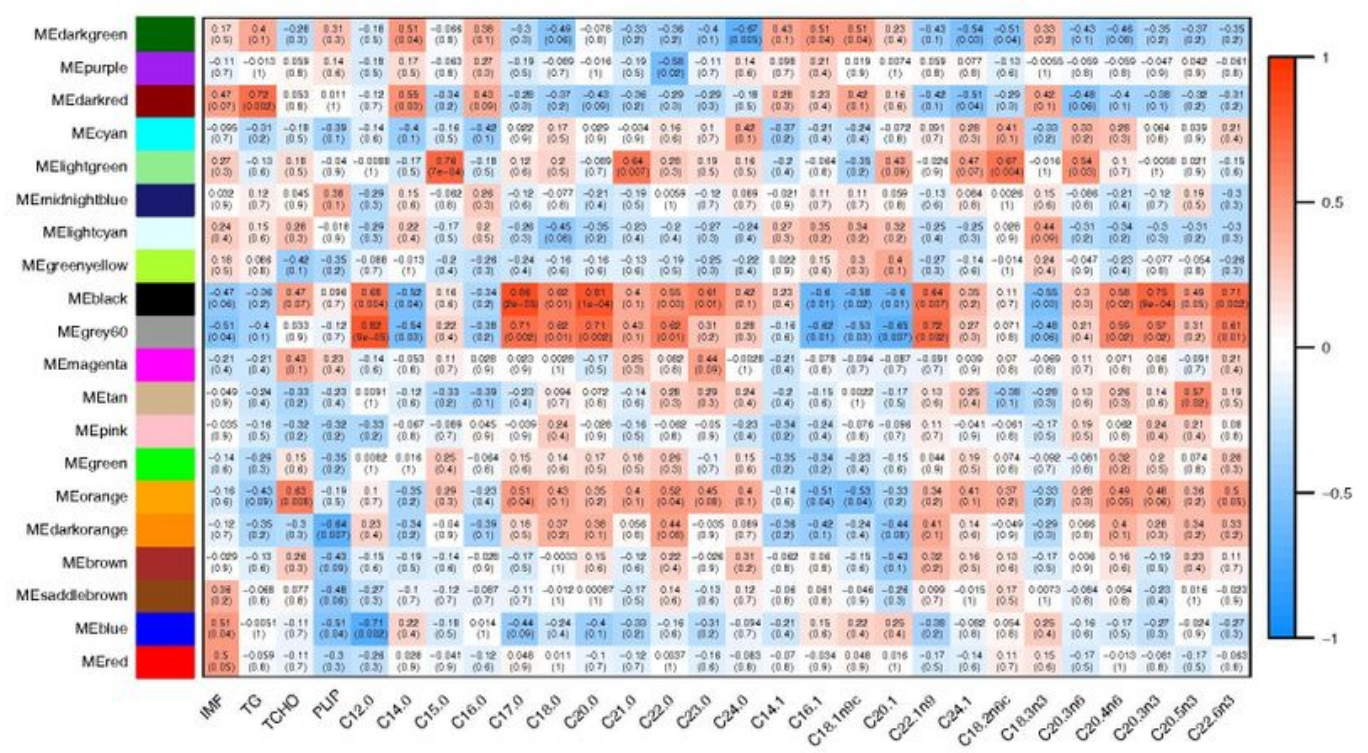

B

Module-stage-trait relationships

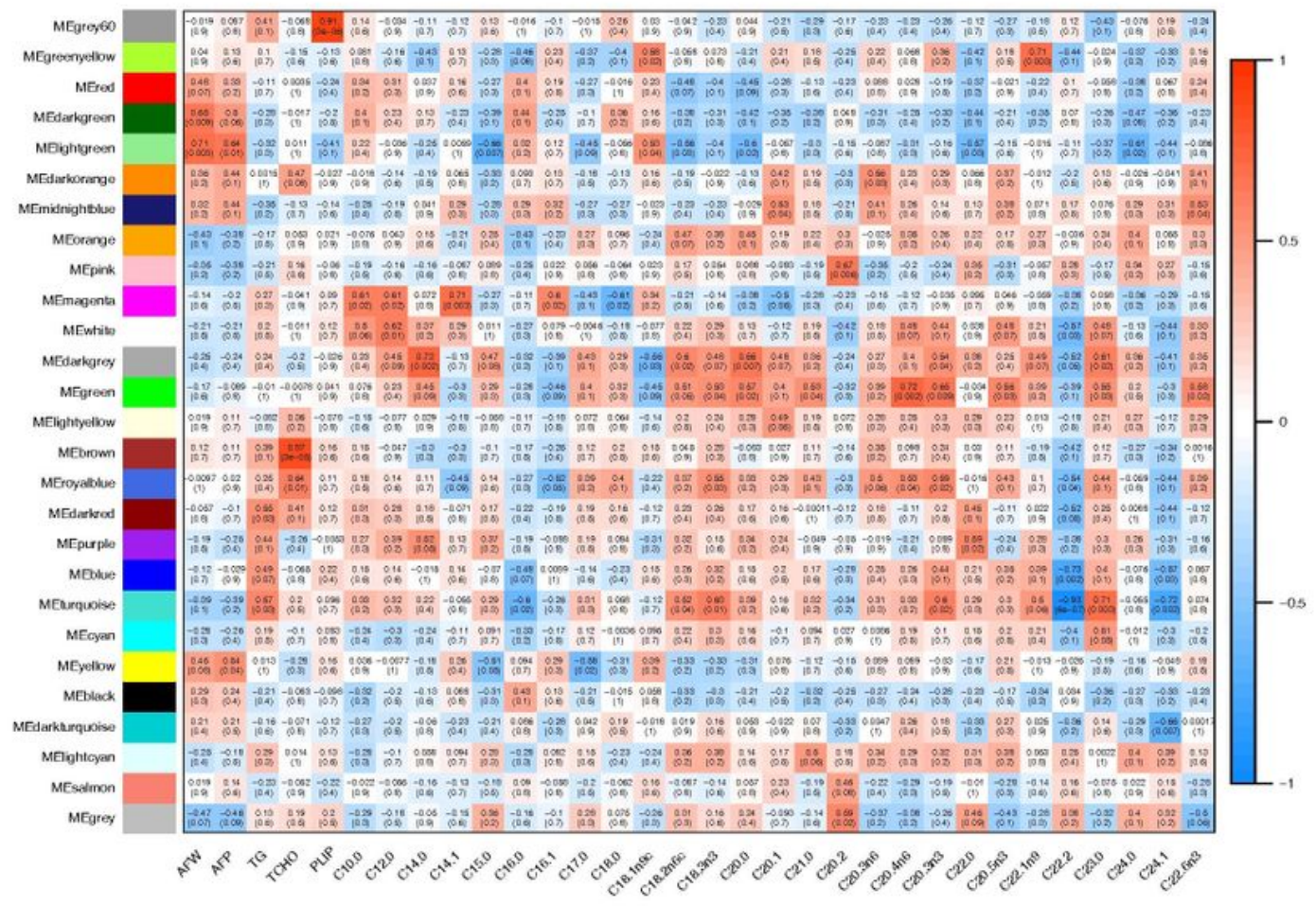

Figure 4 
Relationship between gene modules, triglycerides, and other genetic traits in breast muscle $(A)$ and abdominal fat (B). Note: The upper value in each module is the correlation coefficient between the module and the lower character, and the lower value is the $p$-value of the coefficient.

A

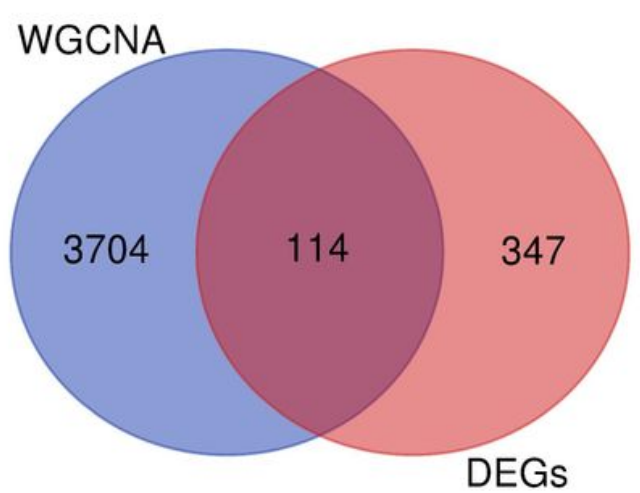

B

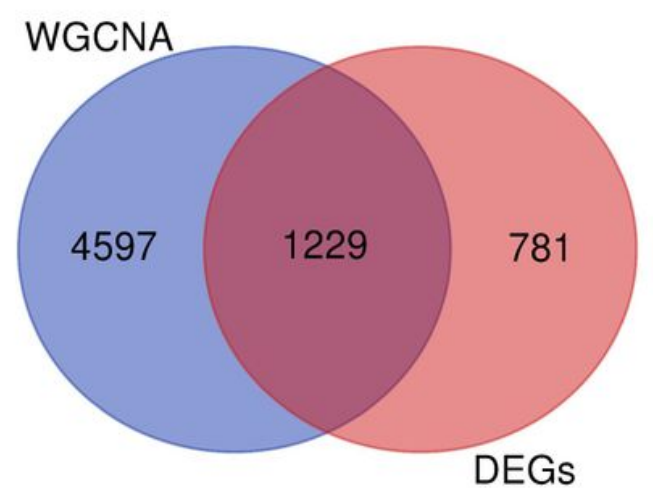

\section{Figure 5}

Venn of module characteristic genes and differential expression genes. (A) Breast muscle tissue (B) Abdominal fat tissue. 


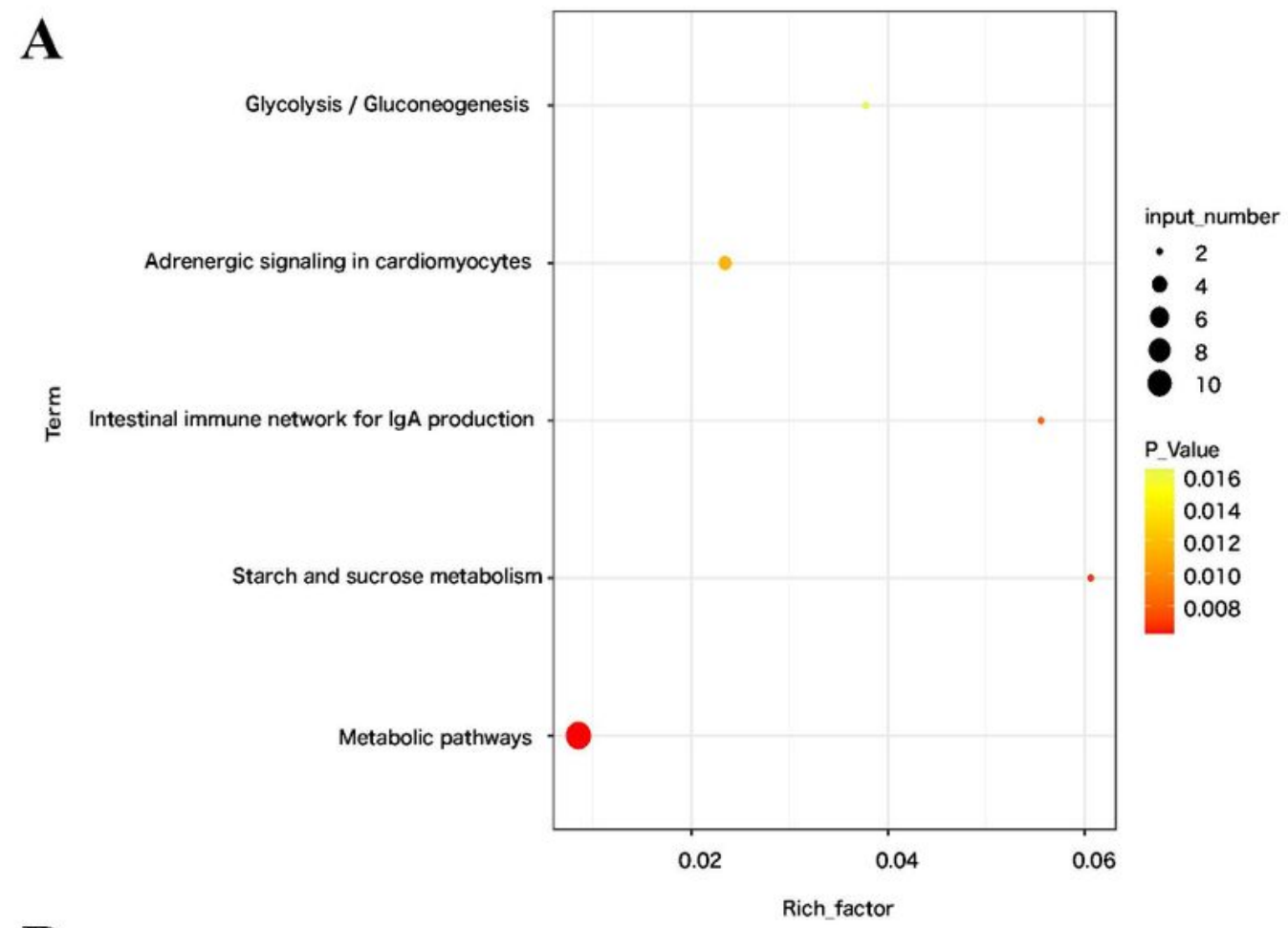

B

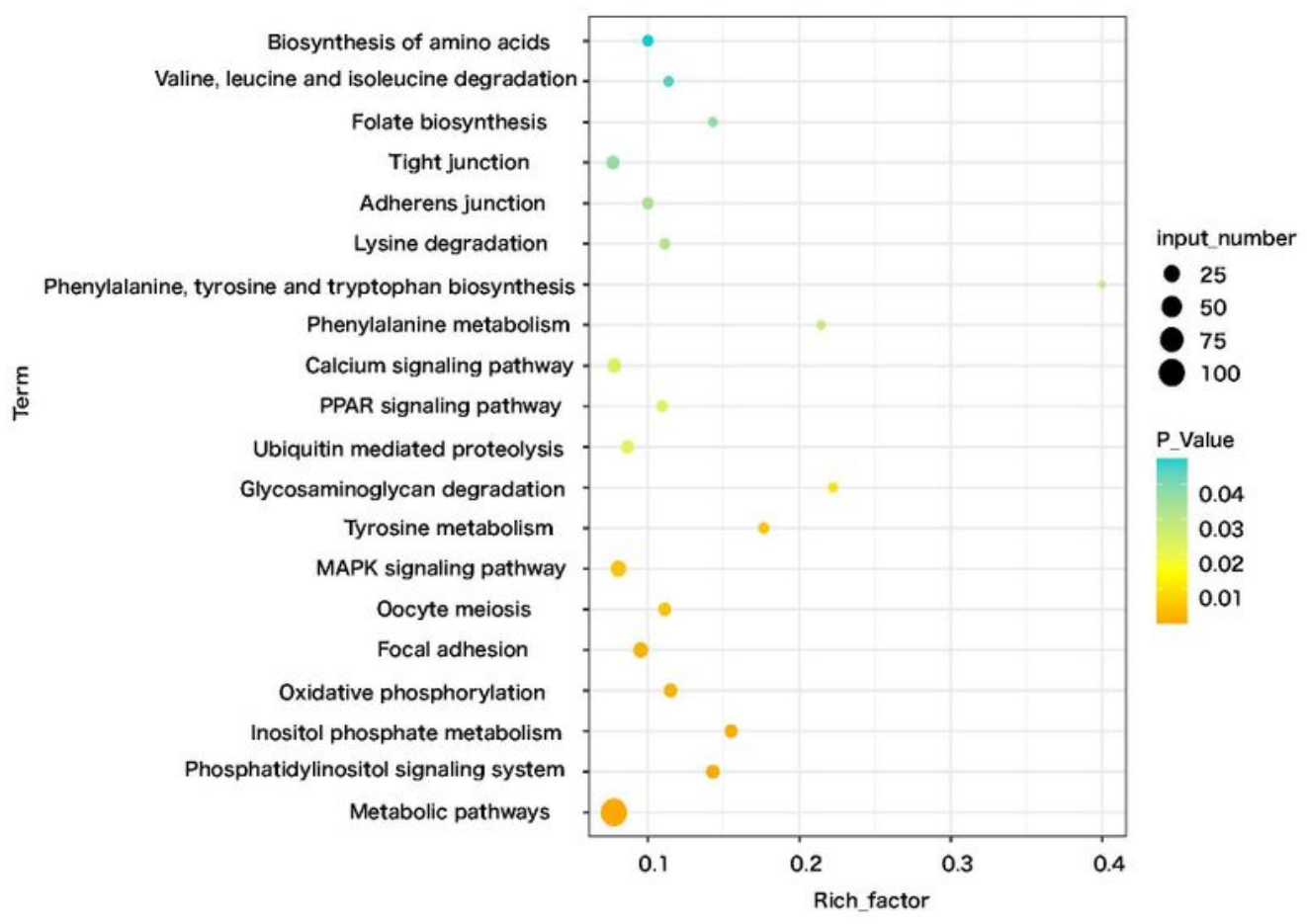

Figure 6

Pathway enrichment analysis of co-expression genes in breast muscle(A) and abdominal fat (B). 
A

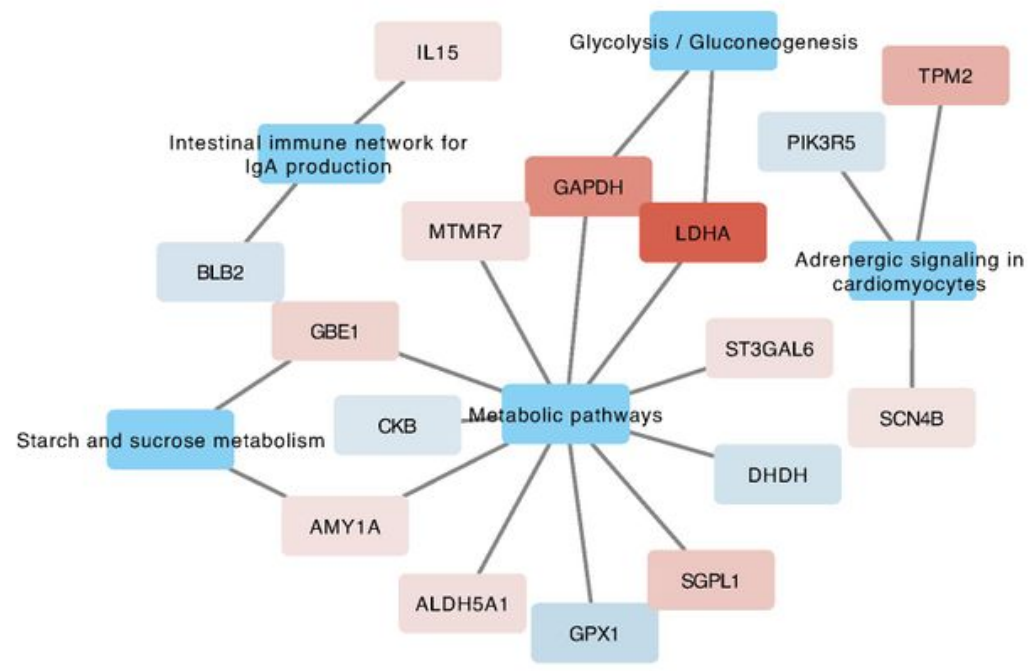

B

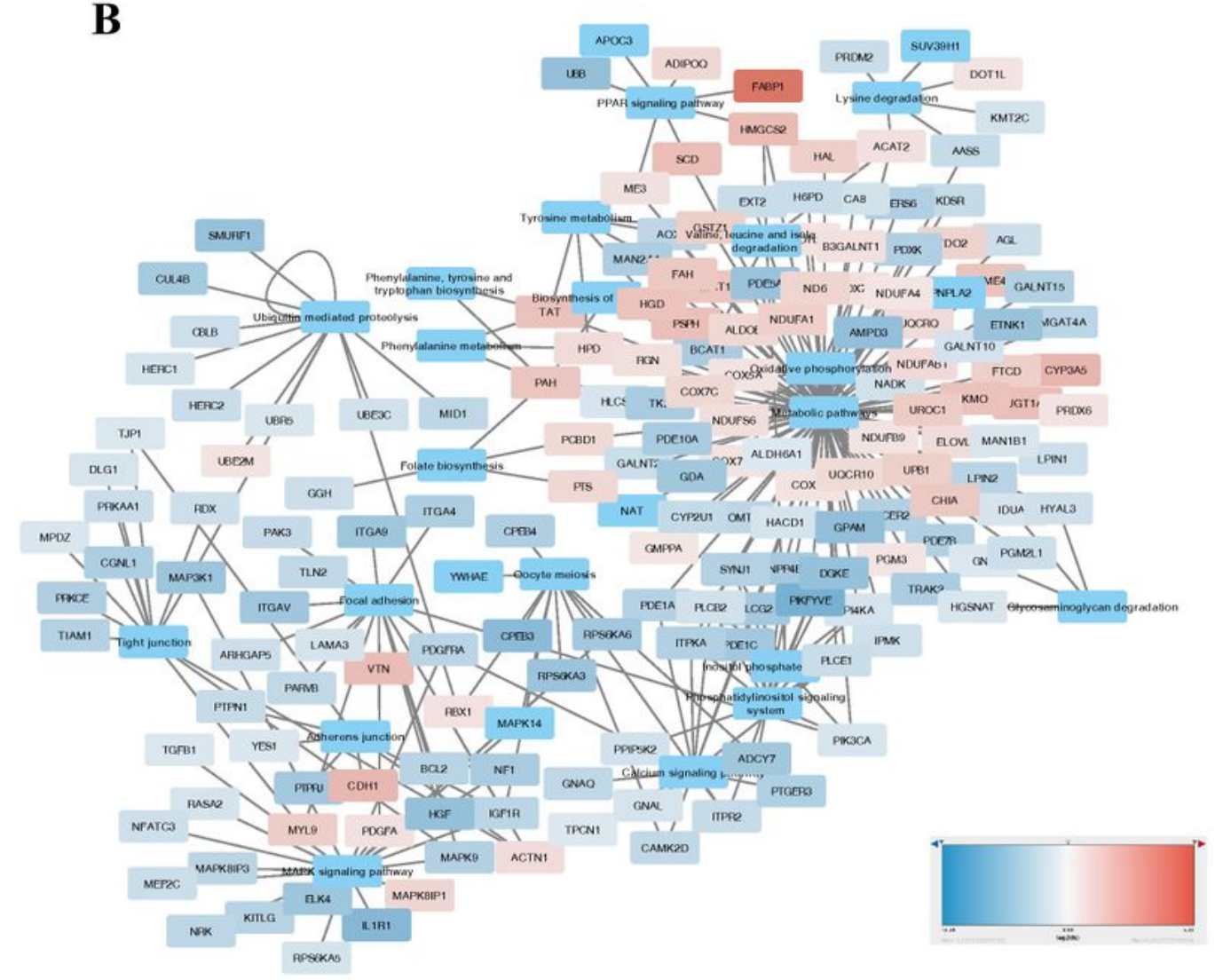

Figure 7

Network of pathways and genes in breast muscle tissue (A) and abdominal fat tissue(B).

\section{Supplementary Files}

This is a list of supplementary files associated with this preprint. Click to download. 
- Additionalfile.xlsx

Page 20/20 